\title{
The Volatility Behavior and Dependence Structure of Commodity Futures and Stocks *
}

\author{
Lin $\mathrm{Gao}^{\dagger \ddagger}$ \\ Lu Liu $\S$
}

forthcoming at the Journal of Futures Markets

${ }^{*}$ We thank Bob Webb (the editor) and an anonymous referee for their helpful comments and suggestions. We are also grateful to Hossein Asgharian, Charlotte Christiansen, Bent Jesper Christensen, Karl Frauendorfer, Pascal Gantenbein, Björn Hansson, Heino Bohn Nielson, Anders Rahbek, Paul Söderlind, Klaus Spremann, and seminar participants at Lund University and the University of St.Gallen, as well as conference participants at the Arne Ryde Workshop in Financial Economics, the 2nd Humboldt-Copenhagen Conference in Financial Econometrics. We thank Hossein Asgharian for his coding of the algorithm of simulated annealing. Financial support from the Bankforskningsinstitutet is appreciated.

${ }^{\dagger}$ Lin Gao is at the School of Finance, University of St. Gallen, St.Gallen, Switzerland.

${ }^{\ddagger}$ Corresponding author, the School of Finance, University of St.Gallen, Rosenbergstr. 52, CH-9000 St.Gallen, Switzerland. Tel.:+41712247074, Fax.:+41712247088, e-mail: lin.gao@unisg.ch.

${ }^{\S} \mathrm{Lu}$ Liu is at the Department of Economics, Lund University, Lund, Sweden. Tel.: +4646222 4290, e-mail:lu. liu@nek. lu. se. 


\begin{abstract}
This paper finds substantial risk diversification potential between certain commodity groups and stocks by exploring the dependence between their patterns of regime switching. None of the commodity groups share a common volatility regime with stocks, nor are the regime switching patterns of grains, industrials, metals, or softs, dependent on that of stocks. Simultaneous volatile regimes of commodity futures and stocks tend to be infrequent and short-lived. In addition, in spite of financial contagion, animal products, grains, and softs typically demonstrate very low correlations with stocks even in simultaneous volatile regimes.
\end{abstract}

\title{
1 Introduction
}

Empirical studies show that financial assets such as stocks and bonds periodically switch from a low volatility regime to a high volatility regime, and then back. Furthermore, assets tend to have larger co-movements with one another in crises despite their low correlations in tranquil periods (see Hartmann et al., 2004). Therefore, it is crucial for investors to understand the periodic regime-switching of financial assets and the dependence structure between the switching processes.

As an alternative investment opportunity, commodity futures returns tend to have low correlations with the returns of traditional assets such as stocks (see Gorton and Rouwenhorst, 2006 and Erb and Harvey, 2006) and are thus an ideal option for portfolio diversification. In the meantime, from a risk management perspective, it is desirable that the regime switching of commodity futures be driven by different (latent) factors from those driving the regime switching of stocks, or more strongly, that the switches of commodity futures be completely unrelated to the (latent) factors driving the switches of stocks. Previous research has not yet paid sufficient attention to such non-linear dependence structures between commodity futures and stocks. Our paper fills this gap.

The present paper employs a bivariate model of switching autoregressive conditional heteroskedasticity (SWARCH), in which asset returns switch between different distributions. This model allows testing several interesting hypotheses on the dependence structure. First, by making hypotheses about the transition probabilities between the regimes of low and high volatility, we examine whether the regime switches (or the Markov chains) of the two assets are the same or are 
unrelated. Moreover, this paper tests whether the conditional correlation tends to vary with the switching process of the stocks or that of the commodity futures.

This study differs from earlier research in three key aspects. First, as far as we know, this is the first study of the regime dependence structure between commodity futures and stocks. Previous studies mainly focus on comovement (e.g., Chong and Miffre, 2010). Moreover, this paper investigates regime changes of both assets in a joint framework with bivariate SWARCH instead of dealing with them in a separate modeling context (e.g., Choi and Hammoudeh, 2010, and Fong and See, 2001) or in a common regime, namely when both are in their volatile regimes at the same time (e.g., Chan et al., 2011). In addition, this regime-dependent analysis of the conditional correlation provides inductive evidence for the diversification potential in the different joint-states of the assets, compared to previous research into dynamic correlation, such as Büyüksahin et al. (2010). The identification of "originator" in the correlation changes - stocks or commodities that lead the correlations - is useful for risk hedging.

\section{Data description and preliminary analysis}

The S\&P 500 Composite Index closing prices and the settlement prices for commodity futures traded on the U.S. exchanges (see Table 1) are collected from Datastream. Log returns are constructed from the time series.

The commodities in the sample provide a very diverse set in terms of such factors as seasonality (e.g., harvest time), country of origin, and perishability. As a result of the diversity, seven rebalanced and equally weighted groups of commodities are formed, based on their natural characteristics (see also Bodie and Rosansky, 1980 and Gorton and Rouwenhorst, 2006): animals, energy, grains, industrial materials (industrials), industrial metals (metals), precious metals (precious m.), and softs. The index compositions and data sources are displayed in Table 1. The sample period spans from January 5, 1979 to April 30, 2010². The data frequency is weekly ${ }^{2}$. The descriptive statistics of the data are depicted in Table 2.

\footnotetext{
${ }^{1}$ As an exception, energy runs from April 8, 1983, and metals run from August 9, 1989, due to the limited data availability.

${ }^{2}$ Besides weekly data we used in our paper, we also estimated regime patterns with both daily and monthly returns. The daily data contains much noise, disturbing the regime-shift
} 
Table 1: Data sources

\begin{tabular}{lllll}
\hline \hline No. & Name & Quotes start & Market & Sector \\
\hline 1 & Frozen Pork Bellies & $5 / 1 / 1979$ & Chicago Mercantile Exchange & Animals \\
2 & Live Cattle & $5 / 1 / 1979$ & Chicago Mercantile Exchange & Animals \\
3 & Lean Hogs & $5 / 1 / 1979$ & Chicago Mercantile Exchange & Animals \\
4 & Feeder Cattle & $5 / 1 / 1979$ & Chicago Mercantile Exchange & Animals \\
5 & Heating Oil & $8 / 4 / 1983$ & New York Mercantile Exchange & Energy \\
6 & Crude Oil & $8 / 4 / 1983$ & New York Mercantile Exchange & Energy \\
7 & Wheat & $5 / 1 / 1979$ & Chicago Board of Trade & Grains \\
8 & Corn & $5 / 1 / 1979$ & Chicago Board of Trade & Grains \\
9 & Soybeans & $5 / 1 / 1979$ & Chicago Board of Trade & Grains \\
10 & Soybean Oil & $5 / 1 / 1979$ & Chicago Board of Trade & Grains \\
11 & Soybean meal & $5 / 1 / 1979$ & Chicago Board of Trade & Grains \\
12 & Oats & $5 / 1 / 1979$ & Chicago Board of Trade & Grains \\
13 & Cotton & $5 / 1 / 1979$ & Coffee, Sugar, and Cocoa Exchange & Industrials \\
14 & Lumber & $5 / 1 / 1979$ & Chicago Mercantile Exchange & Industrials \\
15 & Copper & $1 / 9 / 1989$ & New York Commodities Exchange & Metals \\
16 & Platinum & $5 / 1 / 1979$ & New York Mercantile Exchange & Precious Metals \\
17 & Gold & $5 / 1 / 1979$ & New York Commodities Exchange & Precious Metals \\
18 & Palladium & $5 / 1 / 1979$ & New York Mercantile Exchange & Precious Metals \\
19 & Cocoa & $5 / 1 / 1979$ & Coffee, Sugar and Cocoa Exchange & Softs \\
20 & Sugar & $5 / 1 / 1979$ & Coffee, Sugar and Cocoa Exchange & Softs \\
21 & Orange Juice & $5 / 1 / 1979$ & New York Commodities Exchange & Softs \\
22 & Coffee & $5 / 1 / 1979$ & Coffee, Sugar and Cocoa Exchange & Softs \\
\hline \hline
\end{tabular}

Table 2: Descriptive statistics of the index logarithmic returns

\begin{tabular}{lcccccccc}
\hline \hline & Animals & Energy & Grains & Industrials & Metals & Precious M. & Softs & S\&P 500 \\
\hline Mean & 0.00013 & 0.00034 & 0.00011 & 0.00006 & 0.00039 & 0.00047 & 0.00003 & 0.00066 \\
Std. Dev. & 0.01140 & 0.02062 & 0.01220 & 0.01309 & 0.01579 & 0.01374 & 0.01275 & 0.01001 \\
Kurtosis & 1.60142 & 3.24401 & 2.00160 & 15.48079 & 4.51702 & 7.47411 & 21.11919 & 6.41339 \\
Skewness & 0.03416 & -0.49865 & -0.11862 & -1.18982 & -0.77664 & -0.34063 & 1.28209 & -0.74493 \\
Corr. & 0.07225 & 0.02363 & 0.14817 & 0.18602 & 0.25325 & 0.12613 & 0.07759 & 1 \\
\hline \hline
\end{tabular}

Note: Descriptive statistics for the log-returns of the investigated indexes: mean, standard deviation, excess kurtosis, skewness, and correlation with S\&P 500.

Despite the fact that commodities are traded and consumed worldwide, we restrict our analysis to the U.S. markets for the following reasons. First, the identification with additional spikes. Moreover, from an illustrative perspective, with a long sample period and many data points the regimes estimated with the daily data are quite dense and less persistent. Using the monthly data, the regime division is similar to the weekly data, but the quality of regime identification is inferior to that with the weekly data. 
commodity futures selected are all traded on the U.S. futures exchanges. Second, the U.S. stock market is representative of the world financial markets.

As shown in Table 2, all the time series are leptokurtic. Furthermore, most time series, excluding animals and softs, are negatively skewed. The skewness and the excess kurtosis of the market returns provide further incentives for us to adopt the Markov switching method. ${ }^{3}$ Table 2 also displays the simple correlations with the S\&P 500. Metals has the largest correlation during the sample period, whereas energy has the smallest.

\section{Model and estimation framework}

This study examines the dependence between stocks and commodity futures in a bivariate switching ARCH (SWARCH) modeling framework. Based on the estimations, two types of hypotheses are tested to make in-depth inferences about the dependence structures between stocks and each group of commodity futures.

\subsection{Bivariate SWARCH model (Model A)}

The bivariate SWARCH model we use is a variant of the bivariate SWARCH of Hamilton and Lin (1996) and Edwards and Susmel (2003), which were developed based on the univariate SWARCH of Cai (1994) and Hamilton and Susmel (1994). For the sake of brevity, our bivariate SWARCH model is called Model A. It is

$$
\begin{gathered}
\boldsymbol{r}_{t}=\boldsymbol{\mu}_{s_{t}}+\boldsymbol{u}_{t}, \text { where } \boldsymbol{u}_{t} \mid \boldsymbol{I}_{t-1} \sim N\left(0, \boldsymbol{H}_{t}\right), \\
\boldsymbol{H}_{t}=\left(\begin{array}{cc}
h_{t}^{+} & \rho_{s_{t}} \sqrt{h_{t}^{+} h_{t}^{*}} \\
\rho_{s_{t}} \sqrt{h_{t}^{+} h_{t}^{*}} & h_{t}^{*}
\end{array}\right), \\
h_{t}^{+}=g_{s_{t}^{+}}^{+}\left[a^{+}+b^{+}\left(u_{t-1}^{+}{ }^{2} / g_{s_{t-1}^{+}}^{+}\right)\right], \\
h_{t}^{*}=g_{s_{t}^{*}}^{*}\left[a^{*}+b^{*}\left(u_{t-1}^{*} / g_{s_{t-1}^{*}}^{*}\right)\right] .
\end{gathered}
$$

The superscript + stands for stocks, and $*$, for commodity futures. $r_{t}=\left(r_{t}^{+}, r_{t}^{*}\right)^{\prime}$, and $\mu_{s_{t}}=\left(\mu_{s_{t}^{+}}^{+}, \mu_{s_{t}^{*}}^{*}\right)^{\prime}$, where $r_{t}^{+}$denotes the returns of stocks, and $r_{t}^{*}$ is the commodity futures returns. $\mu_{s_{t}^{+}}^{+}$and $\mu_{s_{t}^{*}}^{*}$ are the respective state-dependent means.

\footnotetext{
${ }^{3}$ Timmermann (2000) theoretically elaborates that Markov switching method alleviates the problems of excess kurtosis and skewness, whereas Li and Lin (2004) provides empirical evidence.
} 
$S_{t}^{+}$is an unobserved state variable that reflects the volatility state of stocks, and $S_{t}^{*}$ represents the volatility state of the individual commodity futures. We assume that each time series switches between two regimes: a low and a high volatility regime. $S_{t}^{+}=1$ and $S_{t}^{*}=1$ denote the low-volatility states of stocks and of commodity futures, while $S_{t}^{+}=2$ and $S_{t}^{*}=2$ denote their high-volatility states. In the bivariate case, there are four combinations of regimes, represented as $S_{t}$.

$$
\begin{aligned}
& S_{t}=1 \text { if } S_{t}^{+}=1 \text { and } S_{t}^{*}=1 \\
& S_{t}=2 \text { if } S_{t}^{+}=2 \text { and } S_{t}^{*}=1 \\
& S_{t}=3 \text { if } S_{t}^{+}=1 \text { and } S_{t}^{*}=2 \\
& S_{t}=4 \text { if } S_{t}^{+}=2 \text { and } S_{t}^{*}=2
\end{aligned}
$$

$S_{t}$ is assumed to follow a first-order Markov chain, with transition probabilities

$$
P\left(S_{t}=j \mid S_{t-1}=i\right)=p_{i j},
$$

for $i, j=1, \ldots, 4$ and $\sum_{j=1}^{4} p_{i j}=1$ for all $j$.

The conditional covariance matrix $\boldsymbol{H}_{t}$ is specified in Equation (2). The conditional variance of the stocks, $h_{t}^{+}$, and the conditional variance of the commodity futures, $h_{t}^{*}$, each follow a SWARCH process. The scaling factors $g_{s_{t}^{+}}^{+}$ and $g_{s_{t}^{*}}^{*}$ measure the scales of the ARCH processes. The factors for the low volatility regimes, $g_{1}^{+}$and $g_{1}^{*}$, are normalized at unity, and both $g_{2}^{+}>1$ and $g_{2}^{*}>1$. The correlation coefficient $\rho$ varies across the four different states ${ }^{4}$.

Following Hamilton (1989), we date the regimes based on their probabilities. First, we use all the sample information to estimate the smoothed probability at time $t$ : $P\left(S_{t} \mid r_{T}, r_{T-1}, \ldots\right)$, using the algorithm of Kim and Nelson (1999). Second, we infer which regime the asset is in at time $t$ using the criterion that the smoothed probability of that regime should be larger than 0.5 .

\subsection{Dependence hypotheses testing (Model B-E)}

The first type of hypothesis regards the regime shift dependence between the Markov chains of the two assets. Model B assumes that the commodity futures

\footnotetext{
${ }^{4}$ Alternatively, we could also adopt time-varying correlations in our model setting. However, in order to provide an inductive intuition for the correlations in the different volatility regimes, we set the correlation coefficient to be constant within each regime. In fact, previous studies document the association of the conditional correlations with the volatility states (e.g., Ramchand and Susmel, 1998).
} 
and stocks share a common pattern of regime switches: $S_{t}^{+}=S_{t}^{*}$. Namely, the states $S_{t}=2$ and $S_{t}=3$ are excluded. In this case, the transition probability matrix is reduced to a $2 \times 2$ matrix.

Model $\mathrm{C}$ assumes that the regime switching pattern of stocks is unrelated to that of commodities, in which case $S_{t}^{*}$ is independent of $S_{t}^{+}$for all $t$. More precisely, the transition probabilities defined in Equation (5) are calibrated as a product of those for the independent chains governing $S_{t}^{+}$and $S_{t}^{*}$. For example,

$$
P\left(S_{t}=4 \mid S_{t-1}=3\right)=P\left(S_{t}^{+}=2 \mid S_{t-1}^{+}=1\right) P\left(S_{t}^{*}=2 \mid S_{t-1}^{*}=2\right)
$$

The second type of hypothesis is about the correlation structure: First, Model $\mathrm{D}$ assumes that the change of correlation is primarily governed by the regime switching of stocks: $\rho_{S_{t}=1}=\rho_{S_{t}=3}$ and $\rho_{S_{t}=2}=\rho_{S_{t}=4}$. In this model, the S\&P 500 is called the "originator." Second, Model E attributes the variation of the correlation to the changes in commodity volatility: $\rho_{S_{t}=1}=\rho_{S_{t}=2}$ and $\rho_{S_{t}=3}=\rho_{S_{t}=4}$. Here, the commodity futures are called the originator.

For both types of hypothesis tests, the general model (Model A described in section 3.1) with unrestricted transition probabilities and correlations serves as the benchmark case. We implement a likelihood ratio test to compare the likelihood of the Model A with that of the restricted model B-E. The likelihood ratio approximately follows a $\chi^{2}$ distribution with $k$ degrees of freedom, where $k$ is the number of additional parameters estimated for the unrestricted model compared to the restricted one.

\section{Empirical analysis}

This section addresses the research questions raised in the first part of the paper. First, we present the results of the general case (Model A). Next, bivariate tests of common (Model B) and independent (Model C) regimes of commodity futures and stocks are implemented. Last, the conditional correlations and the related hypotheses tests are analyzed.

\subsection{Bivariate analysis of the dependence structures}

Table 3 reports the estimated parameters of Model A. For the sake of brevity, the name of each commodity refers to the respective bivariate model with stocks in the following analysis. In contrast to stocks, high volatility is not necessarily associated 
Table 3: Maximum likelihood estimates for the bivariate SWARCH (Model A)

\begin{tabular}{llllllll}
\hline \hline & Animals & Energy & Grains & Industrials & Metals & Precious M. & Softs \\
\hline$\mu_{1}^{+}$ & $0.0014^{* * *}$ & $0.0013^{* * *}$ & $0.0013^{* * *}$ & $0.0013^{* * *}$ & $0.0009^{* * *}$ & $0.0013^{* * *}$ & $0.0014^{* * *}$ \\
$\mu_{2}^{+}$ & -0.0006 & -0.0007 & -0.0005 & $9.2 \mathrm{e}-005$ & 0.0006 & -0.0001 & -0.0004 \\
$\mu_{1}^{*}$ & 0.0001 & $0.0011^{* *}$ & -0.0002 & 0.0001 & 0.0004 & $0.0005^{*}$ & $-5.4 \mathrm{e}-005$ \\
$\mu_{2}^{*}$ & 0.0002 & -0.0031 & 0.0013 & 0.0004 & 0.0009 & 0.0003 & 0.0001 \\
$g_{2}^{+}$ & $3.875^{* * *}$ & $4.017^{* * *}$ & $4.280^{* * *}$ & $3.811^{* * *}$ & $3.832^{* * *}$ & $3.812^{* * *}$ & $3.907^{* * *}$ \\
$g_{2}^{*}$ & $3.591^{* * *}$ & $4.383^{* * *}$ & $4.644^{* * *}$ & $2.339^{* * *}$ & $4.278^{* * *}$ & $6.538^{* * *}$ & $2.971^{* * *}$ \\
$b^{+}$ & $0.160^{* * *}$ & $0.180^{* * *}$ & $0.147^{* * *}$ & $0.150^{* * *}$ & $0.198^{* * *}$ & $0.177^{* * *}$ & $0.152^{* * *}$ \\
$b^{*}$ & $8.1 \mathrm{e}-005$ & 0.045 & $0.070^{*}$ & 0.030 & $7.6 \mathrm{e}-006$ & $0.068^{* *}$ & $1.8 \mathrm{e}-005$ \\
$\rho_{1}$ & 0.0030 & $0.112^{* * *}$ & $0.080^{*}$ & $0.158^{* * *}$ & 0.051 & 0.057 & $0.116^{* *}$ \\
$\rho_{2}$ & $0.188^{* *}$ & -0.194 & 0.136 & -0.069 & $0.151^{* * *}$ & 0.030 & 0.076 \\
$\rho_{3}$ & -0.075 & -0.359 & $0.215^{* *}$ & 0.083 & 0.164 & 0.134 & -0.104 \\
$\rho_{4}$ & $0.201^{*}$ & $0.357^{* * *}$ & $0.200^{* * *}$ & $0.345^{* * *}$ & $0.521^{* * *}$ & $0.302^{* * *}$ & $0.130^{*}$ \\
LogL. & 10449 & 8294 & 10412 & 10324 & 6615 & 10321 & 10368 \\
\hline \hline
\end{tabular}

Note: This table shows the results of the general bivariate SWARCH (Model A) of 4 states composed of 2 states for commodity futures and 2 states for stocks. In the first column, + stands for stocks and ${ }^{*}$ stands for commodity futures. In the other columns, ${ }^{*}$ indicates significance at the $10 \%,{ }^{* *}$ at the $5 \%$ level, and ${ }^{* * *}$ at the $1 \%$ level. Standard errors are in parentheses.

with low or negative average returns in commodity futures. All commodity futures, except energy and precious metals, yield higher returns in their high volatility regimes than in their low volatility regimes. This phenomenon is consistent with the argument in Gorton et al. (2007), in which they observe infrequent upward spikes in the prices of commodity futures, but no downward spikes.

To illustrate the persistence of the regimes, Table 4 reports the empirical frequency and the estimated duration for each state of Model A. The empirical frequency denotes the number of weeks in state $j$ divided by the total number of weeks in the sample period. The duration of state $j$, calculated as $1 /\left(1-p_{j j}\right)$ (Kim and Nelson, 1999), gives how many weeks, on average, regime $j$ lasts. The mutual volatile state (i.e., the fourth state) occupies only $10 \%$ on average of the stockcommodity combinations. Except for metals, the mutual tranquil state (state 1) has the highest empirical frequency ( $54 \%$ on average of the whole sample) among the four states for all the bivariate models. The expected durations of the mutual volatile state are also on average smaller than those of the other states. Animals is 
Table 4: Duration and empirical frequency of the general bivariate SWARCH (Model A)

\begin{tabular}{|c|c|c|c|c|c|c|c|}
\hline & Animals & Energy & Grains & Industrials & Metals & Precious M. & $\overline{\text { Softs }}$ \\
\hline \multicolumn{8}{|c|}{ Duration } \\
\hline state 1 & 57.78 & 79.79 & 14.62 & 24.25 & 107.20 & 61.47 & 15.10 \\
\hline state 2 & 3.57 & 9.63 & 4.99 & 25.62 & 102.73 & 13.33 & 35.58 \\
\hline state 3 & 9.69 & 6.83 & 6.72 & 102.52 & 12.23 & 9.51 & 4.65 \\
\hline state 4 & 1.42 & 15.49 & 13.86 & 24.84 & 26.15 & 4.26 & 13.53 \\
\hline \multicolumn{8}{|c|}{ Empirical frequency } \\
\hline state 1 & $57 \%$ & $64 \%$ & $63 \%$ & $39 \%$ & $41 \%$ & $60 \%$ & $56 \%$ \\
\hline state 2 & $23 \%$ & $14 \%$ & $11 \%$ & $13 \%$ & $47 \%$ & $20 \%$ & $17 \%$ \\
\hline state 3 & $12 \%$ & $11 \%$ & $10 \%$ & $23 \%$ & $4 \%$ & $8 \%$ & $7 \%$ \\
\hline state 4 & $2 \%$ & $7 \%$ & $11 \%$ & $22 \%$ & $9 \%$ & $7 \%$ & $14 \%$ \\
\hline \multicolumn{8}{|c|}{$\begin{array}{l}\text { Note: Expected durations and empirical frequencies of all states for the } \\
\text { general bivariate SWARCH of the commodity futures. The duration of } \\
\text { state } j \text { states how many weeks, on average, one regime } j \text { will last. The } \\
\text { empirical frequency is estimated as the number of weeks that are in state } \\
j \text { divided by the total number of weeks in the sample period. }\end{array}$} \\
\hline
\end{tabular}

Table 5: Likelihood ratio test against the independent and common models (Model B and Model C)

\begin{tabular}{llllllll}
\hline \hline & Animals & Energy & Grains & Industrials & Metals & Precious M. & Softs \\
\hline Likelihood Ratio & $68.66^{* * *}$ & $262.93^{* * *}$ & $78.40^{* * *}$ & $77.79^{* * *}$ & $101.61^{* * *}$ & $67.91^{* * *}$ & $67.62^{* * *}$ \\
Conclusion & uncom & uncom & uncom & uncom & uncom & uncom & uncom \\
\hline \multirow{7}{c}{ Independence (Model C) } \\
Likelihood Ratio \\
Conclusion & $18.69^{* *}$ & $28.33^{* * *}$ & 10.30 & 12.82 & 4.61 & $13.72^{*}$ & 11.83 \\
\hline
\end{tabular}

\footnotetext{
Note: Model B assumes that the commodity futures have the same regimes with stocks. The likelihood ratio is approximately distributed as $\chi_{12}^{2}$, as the unrestricted model A has 12 more parameters than the restricted model $\mathrm{B}$. Model $\mathrm{C}$ assumes that the regime switching patterns of commodity futures and stocks are independent. The likelihood ratio is approximately distributed as $\chi_{8}^{2}$, as the unrestricted model A has 8 more parameters than Model C. *, ** and $* * *$ indicate that the restricted model (null hypothesis) is rejected at, respectively, significance levels of $10 \%, 5 \%$, and $1 \%$.
}

advantageous in risk diversification of a portfolio of stocks, as their mutual volatile state occurs in $2 \%$ of the entire sample period and tends to last for only 1.43 weeks.

Next, the results of the dependence tests of Model B and Model C are reported in Table 5. The null hypothesis that commodity futures and stocks are driven by common latent forces (Model B) is rejected for all commodities at the $5 \%$ level 
of significance. Further, Model C is not rejected for grains, industrials, metals, or softs, implying that the latent driving factors of these commodity futures are independent of the latent factors driving stocks. Dependence is found only in energy and precious metals, which are known to be closely related to stock markets, and in animals - the only pure domestic products in our sample. The non-mutual and independent regime switches between most commodities and stocks favors the diversification between these two kinds of assets.

To summarize, the results detect three attractive properties of commodity futures that are favorable for risk diversification in a portfolio. First, the mutual volatile regimes of commodity futures and stocks tend to be infrequent and short-lived. Second, commodity futures tend to be subject to upward instead of downward price changes. Finally, the conditional variances of stocks and commodities are not necessarily subject to the same or interdependent driving factors, as manifested in the independent and uncommon regime identification between them.

\subsection{Correlation tests and analysis}

In addition to the dependence of regime switching patterns, correlations conditioned on various regimes also play a key role in asset diversification. The bottom part of Table 3 displays the conditional correlation coefficient $\rho$ for the general model (Model A). The small value of $\rho$ over all the regimes favors diversification between stocks and commodity futures. $\rho$ in the fourth regime (i.e., when both stock markets and commodity futures are volatile) is larger than those in the other regimes for all groups except for grains. However, the value of $\rho$ in the fourth regime is still small in all cases except for metals, suggesting that the benefit of diversification prevails. Despite the high correlation (0.521) between metals and stocks in the fourth regime, this regime takes up only $9 \%$ of the sample period (see the empirical frequency in Table 4). The lowest correlation in the fourth regime is found between softs and stocks (0.130).

As a comparison with commodity futures, we consider the diversification potential between the world stock index (excluding the U.S. market) from Datastream and the S\&P 500 as a benchmark. Table 6 presents the estimated duration, empirical frequency, and correlations between these two series using 
Table 6: Selected estimation results of the bivariate model A between the world stock index (excluding the U.S. market) and the S\&P 500 index

\begin{tabular}{lcccc}
\hline \hline & State 1 & State 2 & State 3 & State 4 \\
\hline Duration & 24.17 & 1.01 & 6.08 & 10.13 \\
Empirical Frequency & $61 \%$ & $7 \%$ & $9 \%$ & $23 \%$ \\
$\rho$ & $0.541^{* * *}$ & $0.462^{* * *}$ & 0.039 & $0.717^{* * *}$ \\
\hline \hline
\end{tabular}

Note: Selected results of the bivariate SWARCH between world stock index (excluding the U.S. market) and S\&P 500 index. Duration, empirical frequency, and correlation are shown for each state. Standard errors of $\rho$ are in parentheses. ${ }^{* * *}$ denotes the $1 \%$ significance level.

Table 7: Likelihood ratio test against Model D and Model E

\begin{tabular}{llllllll}
\hline \hline & Animals & Energy & Grains & Industrials & Metals & Precious M. & Softs \\
\hline \multicolumn{7}{c}{} & \multicolumn{7}{c}{ Model D } \\
$\rho_{1}=\rho_{3}$ & -0.008 & $0.087^{* *}$ & $0.115^{* * *}$ & $0.104^{* * *}$ & 0.067 & 0.025 & $0.067^{*}$ \\
$\rho_{2}=\rho_{4}$ & 0.175 & -0.063 & $0.168^{* * *}$ & $0.259^{* * *}$ & $0.242^{* * *}$ & $0.188^{* * *}$ & $0.090^{*}$ \\
Likelihood Ratio & 0.48 & $14.17^{* * *}$ & 2.77 & $6.19^{* *}$ & $9.54^{* * *}$ & $4.73^{*}$ & 3.36 \\
\hline \multicolumn{7}{c}{ Model E } \\
$\rho_{1}=\rho_{2}$ & 0.036 & 0.015 & $0.095^{* * *}$ & $0.139^{* * *}$ & $0.116^{* * *}$ & $0.056^{*}$ & $0.090^{* *}$ \\
$\rho_{3}=\rho_{4}$ & 0.084 & 0.020 & $0.208^{* * *}$ & $0.214^{* * *}$ & $0.449^{* * *}$ & $0.237^{* * *}$ & 0.050 \\
Likelihood Ratio & $7.92^{* *}$ & $17.91^{* * *}$ & 0.45 & $10.15^{* * *}$ & $5.02^{*}$ & 1.37 & 3.18 \\
\hline Originator & stocks & neither & both & neither & neither & commodity & both \\
\hline \hline
\end{tabular}

Note: Estimated correlations between commodity futures and S\&P 500 of Model D and Model E. Also shown are the results of the likelihood ratio tests for the general bivariate SWARCH (Model A) against Model D and E. The likelihood ratio is approximately distributed as $\chi_{2}^{2}$, as Model A has 2 more parameters than Model D and E. ${ }^{* * *},{ }^{* *}$, and ${ }^{*}$ denote null hypothesis rejected at, respectively, the $1 \%, 5 \%$, and $10 \%$ significance levels.

the bivariate model A over the same sample period. The results show that the correlations between the S\&P 500 and the non-US world stock index are significantly positive and are much larger than those with commodity futures in states 1,2, and 4. Although the correlation for state 3 is close to zero, the diversification benefit is almost negligible given the short duration (6.08 weeks) and small empirical frequency $(9 \%)$ of this state. In addition, the hypothesis of independence (Model B) between the S\&P 500 and the non-US world stock index is rejected. Therefore, we conclude that commodity futures are superior to the world stock index in terms of diversification with the U.S. stock market. 
Furthermore, two hypotheses regarding the change in the correlations (Model D and Model E in Section 3.2) are tested. Model D assumes that the S\&P is the "originator" (i.e., the correlations change when stocks switch from one regime to the other), whereas Model E assumes that commodity futures are the originator. Table 7 documents the test results for both hypotheses. The correlations become larger when the stock market turns volatile in all cases except for energy. However, the likelihood ratios demonstrate that only the correlations of animals, grains, and softs are associated with the volatility-regime switches of stocks. The likelihood ratio test shows that Model $\mathrm{E}$ is not rejected for grains, precious metals, or softs. The correlation coefficient between precious metals and the S\&P 500 quadruples, as precious metals turn from their tranquil to their volatile state. In addition, both models are rejected for energy, industrial materials, and metals, suggesting that the correlation changes are not aligned with either side of the assets.

\section{Conclusion}

The main finding of this paper is that commodity futures can be a good instrument for risk diversification. They do not share common volatility regimes with U.S. stocks, which is in line with the segmented market view. Furthermore, regimeswitching dependence is only found in energy and precious metals, which are known to be closely related to the stock market, and in animals. The mutual volatile regime of commodity futures and stocks tend to be infrequent and short-lived.

The correlations between the U.S. stock returns and the commodity futures are generally much lower than the correlation between the U.S. and the world (excluding U.S.) stock index returns. Correlations between the U.S. stocks and the commodity futures do increase in periods in which both are volatile. However, the magnitude of the increase is very mild. Moreover, the short duration of the mutual volatile regimes also relieves the undesired effect of the temporary high correlation. In addition, the correlation changes of energy, industrials, and metals with stocks are not dominated by one-sided volatility state changes. In other words, the correlations between stocks and energy may not change dramatically, when only stocks or energy switch from one to the other volatility state. Overall, the results of this paper support risk diversification between commodity futures and stocks. 


\section{References}

Bodie, Z. and Rosansky, V. I. (1980). Risk and return in commodity futures. Financial Analysts Journal, 36(3):27-31+33-39.

Büyüksahin, B., Haigh, M., and Robe, M. (2010). Commodities and equities: ever a "market of one"? Journal of Alternative Investments, 12(3):76-95.

Cai, J. (1994). A Markov model of switching-regime ARCH. Journal of Business E6 Economic Statistics, 12(3):309-316.

Chan, K., Treepongkaruna, S., Brooks, R., and Gray, S. (2011). Asset market linkages: Evidence from financial, commodity and real estate assets. Journal of Banking \& Finance, 35(6).

Choi, K. and Hammoudeh, S. (2010). Volatility behavior of oil, industrial commodity and stock markets in a regime-switching environment. Energy Policy, 38(8):4388-4399.

Chong, J. and Miffre, J. (2010). Conditional correlation and volatility in commodity futures and traditional asset markets. Journal of Alternative Investments, 12(3):61 - 75 .

Edwards, S. and Susmel, R. (2003). Interest-rate volatility in emerging markets. Review of Economics and Statistics, 85(2):328-348.

Erb, C. B. and Harvey, C. R. (2006). The strategic and tactical value of commodity futures. Financial Analysts Journal, pages 69-97.

Fong, W. and See, K. (2001). Modelling the conditional volatility of commodity index futures as a regime switching process. Journal of Applied Econometrics, 16(2):133-163.

Gorton, G., Hayashi, F., and Rouwenhorst, K. (2007). The fundamentals of commodity futures returns. NBER Working Paper 13249.

Gorton, G. and Rouwenhorst, K. (2006). Facts and fantasies about commodity futures. Financial Analysts Journal, 62(2):47-68.

Hamilton, J. (1989). A new approach to the economic analysis of nonstationary time series and the business cycle. Econometrica, 57(2):357-384.

Hamilton, J. and Lin, G. (1996). Stock market volatility and the business cycle. Journal of Applied Econometrics, 11(5):573-593. 
Hamilton, J. and Susmel, R. (1994). Autoregressive conditional heteroskedasticity and changes in regime. Journal of Econometrics, 64(1-2):307-333.

Hartmann, P., Straetmans, S., and Vries, C. (2004). Asset market linkages in crisis periods. Review of Economics and Statistics, 86(1):313-326.

Kim, C. and Nelson, C. R. (1999). State-space models with regime switching, Cambridge, MA: MIT Press.

Li, M. and Lin, H. (2004). Estimating value-at-risk via Markov switching ARCH models-an empirical study on stock index returns. Applied Economics Letters, 11(11):679-691.

Ramchand, L. and Susmel, R. (1998). Volatility and cross correlation across major stock markets. Journal of Empirical Finance, 5(4):397-416.

Timmermann, A. (2000). Moments of Markov switching models. Journal of Econometrics, 96(1):75-111. 